\title{
Study on the Internal Nursing Care of 74 Patients with Upper Gastrointestinal Bleeding
}

\author{
Xiaohong Song* \\ The People's Hospital of Lintao County, Dingxi, Gansu 730500, China
}

\begin{abstract}
Objective: To study the reasonable nursing methods of upper gastrointestinal bleeding by summarizing internal nursing care experience. Methods: Seventy four cases of patients with upper gastrointestinal bleeding from 2014 to 2015 in our hospital were selected. All cases were divided into two groups. The control group: 37 cases were treated with the conventional nursing; the experimental group: 37 cases were treated with conventional nursing and strengthening psychological intervention as well as health education. Differences between the two groups in anxiety, depression index before and after nursing and nursing satisfaction were used to evaluate the nursing effect of the two groups of patients. Results: On the basis of the control group, the experimental group had an SAS score $(39.67 \pm 3.75)$ and an SDS score $(41.35 \pm 2.59)$. The control group had an SAS score (52.34 \pm 2.25$)$ and an SDS score (51.14 \pm 1.38$)$. Different results between the two groups were significant in Statistics, $p<0.05$; moreover, 32 cases were satisfied in the experimental group, the nursing satisfaction was $86.49 \%$ and 26 cases were satisfied in the control group, the nursing satisfaction was 70.27\%. Different results between the two groups were significant in Statistics, $p<0.05$. Conclusion: On the basis of conventional nursing, it was helpful to promote the patients' physical and mental health by strengthening psychological intervention and health education of disease related knowledge; it was worthy of clinical promotion.
\end{abstract}

\section{KEYWORDS}

Gastrointestinal bleeding

High quality nursing

Nursing effect

\section{Introduction}

Upper gastrointestinal bleeding refers to bleeding of gastrointestinal above Treitz, including bleeding in esophagus, stomach and duodenum, pancreatic duct and hemobilia. The causes of upper gastrointestinal bleeding include stomach and duodenum disease, esophageal varices bleeding and portal hypertensive gastropathy caused by portal hypertension, upper gastrointestinal adjacent organs or tissue disease [1]. Upper gastrointestinal bleeding was frequently noted as acute massive bleeding and serious in a critical condition, especially on patients in advanced age or with related complications. Timely and effective therapeu-

Copyright ( 2015 Xiaohong Song

doi: $10.18686 /$ jn.v4i4.1

Received: September 16, 2015; Accepted: November 4, 2015; Published online: December 29, 2015

This is an open-access article distributed under the terms of the Creative Commons Attribution Unported License (http://creativecommons.org/ licenses/by-nc/4.0/), which permits unrestricted use, distribution, and reproduction in any medium, provided the original work is properly cited.

${ }^{*}$ Corresponding author: The People's Hospital of Lintao County, Dingxi, Gansu 730500, China. E-mail: dr_xhs22@gmail.com tic nursing was the key to rescue the lives [2]. High quality nursing was helpful to the recovery of patients with upper gastrointestinal bleeding. In order to study the reasonable nursing methods, the author reported 74 cases of patients with upper gastrointestinal bleeding from 2010 to 2011 in our hospital.

\section{Data \& methods}

\subsection{Data selected}

Seventy four cases of patients with upper gastrointestinal bleeding from 2014 to 2015 in our hospital were selected: 42 men and 32 women from 25-76 years old (mean age $45.5 \pm 5.5$ ); causes: 42 cases with gastrointestinal disease, 28 cases with portal hypertension disease and four cases with hemobilia. All cases were divided into the experimental group (37 cases) and the control group (37 cases). Differences between the two groups were not significant in gender, age and cause; they can be compared, $p>0.05$, see Table 1.

\subsection{Clinical manifestation}

Seventy four cases all had different degrees of clinical 
Table 1. Two groups of patients in general situation.

\begin{tabular}{cccccccc}
\hline \multirow{2}{*}{ Group } & \multicolumn{2}{c}{ Gender } & \multirow{2}{*}{ Age } & & \multicolumn{2}{c}{ Cause } \\
\cline { 2 - 3 } & & Men & Women & & Stomach \& intestine & Portal hypertension & hemobilia \\
\hline Experimental Group & 21 & 16 & $46.4 \pm 6.2$ & 21 & 214 & 14 & 2 \\
Control Group & 21 & 16 & $44.7 \pm 4.3$ & 21 & 2 \\
\hline
\end{tabular}

Note: Differences of general data between the two groups were not significant in Statistics, but comparable, $p>0.05$.

manifestation of upper gastrointestinal bleeding such as hematemesis, melena, fever, elevated hemogram, and hemorrhagic shock. Forty two cases with gastrointestinal disease had epigastric pain, 28 cases with portal hypertension disease had spider angioma and four cases with hemobilia had jaundice.

\subsection{Methods}

According to the different primary diseases, all cases must stanch in different ways actively and control the primary disease. Patients with ulceration had endoscopic hemostasis performed and given anti acid, anti-helicobacter pylori treatment. Patients with cirrhosis and portal hypertension banded the varication in esophagus fundus ventricularis on-time. Patients with hemobilia should control infections.

\subsubsection{Nursing care methods in the control group}

Patients in this group were treated with conventional nursing, including:

\section{General nursing care}

On admission, personal information was recorded and vital signs (including BP, pulse, respirations) were supervised. After admission, the patient was asked to lie on the back to prevent suffocation while spitting blood. By closely observing the change in patients' condition, unobstructed respiratory tract and the effective venous channel were established at the same time. Meanwhile, effective circulating blood volume was recovered and oxygen therapies to protect ischemia organs were administered.

\section{Diet nursing care}

Patients with acute massive bleeding must not eat anything in 24-48 hours to prevent stimulating the gastrointestinal and causing bleeding again. After bleeding, patients should consume less liquid in their diet and avoid hard and cellulose-high content food. Patients with TPN took aseptic operation strictly.

\section{Symptom nursing care}

Hematemesis and melena were the common symptoms in upper gastrointestinal bleeding. When patients spit blood, put the head to one side and cough out blood sputum instead. Clean the blood and apply erythromycin ointment around anus quickly to prevent infection, and keep bowel movement regular.

\section{Infection prevention}

After bleeding in the upper gastrointestinal tract, the patient is vulnerable in breathing in infection into the respiratory system. The reason is that after spitting, there is plenty of blood in the mouth, which provides an encouraging environment for bacteria to grow. So it was necessary to ask patients to gargle with saline, which reduced the respiratory infection. For bedridden patients, it was essential to keep the skin dry, turn over regularly and massage the compression site to avoid the occurrence of bedsores.

\subsubsection{Nursing methods in the experimental group}

The experimental group was given high quality nursing on the basis of the control group, as follows:

\section{Psychological intervention}

Medical staff communicated with patients actively to make themselves trusted; they introduced the illness and treatment to patients' promptly in order to eliminate patients' anxiety, encouraging them to overcome the disease. They should also make a homely environment, keeping a dignified appearance while being strict, yet gentle, and leading patients towards relaxation by listening to music and breathing deeply [3].

\section{Health education}

All cases were given health education on upper gastrointestinal bleeding through lectures. Health education contents include causative factors, inducing factors and nursing knowledge. It is also necessary to introduce the inducing factors in daily life, such as drinking and using nonsteroidal anti-inflammatory drugs, etc [4]. So patients were advised to give up on smoking and drinking. They were also told to follow the advice of medical staff in the prevention of the illness.

\subsection{Evaluation criteria}

SAS and SDS were taken to evaluate mental states before and after nursing in the two groups during one week. There were 20 questions with the score $(0-100)$ respectively. The results divided into 50 to 59 for mild depression or anxiety, 60-69 for severe depression or anxiety, more than 70 points for major depression or anxiety and 50 as boundary value.

\subsection{Statistical methods}

Statistical software SPSS 16.0 was used to analyse and eval- 
uated the observed data.

\section{Results}

On the basis of the control group, the experimental group had an SAS score $(39.67 \pm 3.75)$ and an SDS score (41.35 \pm 2.59). The control group had an SAS score (52.34 \pm 2.25$)$ and an SDS score (51.14 \pm 1.38$)$.Different results between the two groups were significant in Statistics, $p<0.05$, see Table 2. Moreover, 32 cases were satisfied in the experimental group, the nursing satisfaction was $86.49 \%$ and 26 cases were satisfied in the control group, the nursing satisfaction was $70.27 \%$. Different results between the two groups were significant in Statistics, $p<0.05$ (Table 3).

Table 2. Compared SAS and SDS $(\bar{x} \pm s)$ in two groups.

\begin{tabular}{ccc}
\hline Group & Experimental Group & Control Group \\
\hline Event & Before nursing After nursing & Before nursing After nursing \\
SAS & $58.52 \pm 2.3339 .67 \pm 3.75$ & $59.12 \pm 3.4552 .34 \pm 2.25$ \\
SDS & $59.74 \pm 2.3541 .35 \pm 2.59$ & $58.95 \pm 4.3851 .14 \pm 1.38$ \\
\hline
\end{tabular}

Note: SAS and SDS $(\bar{x} \pm s)$ in the experimental group were more obvious than those in the control group. Different results between the two groups were significant in Statistics, $p<0.05$.

Table 3. Nursing Satisfaction [n (\%)].

\begin{tabular}{ccccc}
\hline Group & Satisfied & $\begin{array}{c}\text { General } \\
\text { Satisfied }\end{array}$ & $\begin{array}{c}\text { Unsat- } \\
\text { isfied }\end{array}$ & $\begin{array}{c}\text { Satisfac- } \\
\text { tion }\end{array}$ \\
\hline $\begin{array}{c}\text { Experimental Group } \\
\text { (number) }\end{array}$ & 18 & 14 & 5 & $32(86.49)$ \\
$\begin{array}{c}\text { Control Group (num- } \\
\text { ber) }\end{array}$ & 13 & 13 & 11 & $26(70.27)$ \\
\hline
\end{tabular}

Note: Nursing Satisfaction in the experimental group was obviously higher than that in the control group. Different results between the two groups were significant in Statistics, $p<0.05$.

\section{Discussion}

Upper gastrointestinal bleeding was often an acute onset. Its main clinical manifestation was heavy bleeding. At this time, the patients had more fragile psychology and were easily losing confidence in the treatment of tension. As a result, they might have failed the treatment. When nursing staff were at work, they should communicate with patients and know their psychological situation by offering them sound psychological counselling. They could help patients eliminate negative emotions and overcome the disease. Health education was an important part in modern nursing and it was also a crucial index in evaluating the nurs- ing level. So health education is helpful in recovering and preventing bleeding again $[5,6]$.

Seventy four cases selected were divided into the control group (37 cases) and experimental group (37 cases). On the basis of the control group, the experimental group had the SAS score $(39.67 \pm 3.75)$ and the SDS score (41.35 $\pm 2.59)$. The control group had the SAS score (52.34 \pm $2.25)$ and the SDS score (51.14 \pm 1.38$)$. Different results between the two groups were significant in Statistics, $p<$ 0.05; moreover, 32 cases were satisfied in the experimental group, the nursing satisfaction was $86.49 \%$ and 26 cases were satisfied in the control group, the nursing satisfaction was $70.27 \%$. Different results between the two groups were significant in Statistics, $p<0.05$. The mental state of the patients in the experimental group was significantly better than that in the control group, and the patients' satisfaction with nursing service was higher.

By all accounts, if nursing staff strengthened psychological intervention and health education, it will be helpful in improving nursing satisfaction and the metal situation. Health education on disease was helpful to reduce and eliminate the inducing factors to improve life quality. Psychological intervention helped eliminate bad psychological factors and establish the confidence to overcome the disease. In summary, on the basis of conventional nursing, it was useful to promote the patients' physical and mental health by strengthening psychological intervention and health education on the disease. It was worthy of clinical promotion.

\section{References}

1. Lu Z, Zhong N, Xie Y, et al. Internal Medicine. People's Medical Publishing House. 2010;6(52):483-485.

2. Wei R, Song Y. Application of high quality care in clinical nursing and its effect evaluation. Chinese Journal of Practical Nursing. 2012;28(10):1-2.

3. Zhu X. Applied research of health education on the upper gastrointestinal hemorrhage. Contemporary Medicine. 2009;15(12):22-25.

4. Xin S. A brief analysis of the psychological characteristics and psychological nursing of medical patients. China Healthcare Frontiers. 2009;(8):77.

5. Zhang $X$, Wen $Y$, Zhong $C$. Practice and experience of creating high quality nursing service demonstration wards. Chinese General Nursing. 2011;9(8):146-147.

6. Chen Z, Chen L. Investigating the effect of good care on nursing quality. Journal of Qiqihaer University of Medicine. 2011;32(9):1485-1486. 